PASSOS FA; TRANI PE; CARVALHO CRL. 2015. Desempenho agronômico de genótipos de morangueiro. Horticultura Brasileira 33: 267-271. DOI http://dx.doi.org/10.1590/S0102-053620150000200021

\title{
Desempenho agronômico de genótipos de morangueiro
}

\author{
Francisco A Passos; Paulo E Trani; Cássia RL Carvalho \\ IAC, C. Postal 28, 13012-970 Campinas-SP; fapassos@iac.sp.gov.br; petrani@iac.sp.gov.br; climonta@iac.sp.gov.br
}

\begin{abstract}
RESUMO
Escassas são as informações sobre o comportamento de cultivares de morangueiro (Fragaria $\mathrm{x}$ ananassa), com vistas ao cultivo em São Paulo. Desse modo, o objetivo do trabalho foi avaliar o desempenho agronômico de genótipos de morangueiro em região com potencial para a agricultura periurbana. Foram avaliadas as cultivares Aleluia, Camarosa, Camino Real, Festival, Oso Grande, Tudla e Ventana. O delineamento experimental foi em blocos casualizados, com três repetições, sendo as parcelas constituídas de 14 plantas, quatro delas utilizadas como bordadura, no espaçamento de 40 x $35 \mathrm{~cm}$. Foram avaliadas produção por planta e porcentagem de frutos, referentes a diversas classes de massa fresca, número de frutos por planta e massa do fruto no período inicial (junho-julho) e total de colheitas (junho-outubro) e os teores de sólidos solúveis (SS), acidez titulável (AT), relação SS/AT e de vitamina C. 'Ventana' destacou-se pela maior produção de frutos, sem diferir de 'Festival' e 'Aleluia', inclusive com expressiva produção de frutos de massa acima de $30 \mathrm{~g}$, de maior valor comercial. 'Camino Real' apresentou baixa produção. A melhor relação SS/AT foi apresentada por 'Aleluia', 'Festival', 'Oso Grande' e 'Ventana'. Todas as cultivares apresentaram altos teores de vitamina C. 'Aleluia' e 'Ventana' podem ser consideradas opções vantajosas para o cultivo do morangueiro em São Paulo.
\end{abstract}

Palavras-chave: Fragaria x ananassa, produção, características químicas do fruto.

\begin{abstract}
Agronomic performance of strawberry genotypes

Little information is available about the performance of strawberry cultivars (Fragaria x ananassa) for cultivation in São Paulo state, Brazil. We evaluated the agronomic performance of strawberry cultivars (Aleluia, Camarosa, Camino Real, Festival, Oso Grande, Tudla and Ventana) in a region with potential for peri-urban agriculture. The experimental design was randomized complete blocks, with 3 replications, the plots being composed by 14 plants in the spacing of $40 \times 35 \mathrm{~cm}$. We evaluated yield per plant and percentage of fruits according to fruit mass classes, number of fruits per plant and fruit mass in the early period (June-July) and total of harvests (June-October) and soluble solids (SS), titratable acidity (AT), SS/AT ratio and vitamin C content. 'Ventana' presented the highest yield per plant, without differing from 'Festival' and 'Aleluia', including outstanding yield of fruits weighing more than $30 \mathrm{~g}$ of higher commercial value. 'Camino Real' presented low yield. The best SS/AT ratios were presented by 'Aleluia', 'Festival', 'Oso Grande' and 'Ventana'. All the cultivars presented high vitamin C contents. 'Aleluia' and 'Ventana' can be considered good options for strawberry growing in São Paulo state.
\end{abstract}

Keywords: Fragaria x ananassa, yield, chemical traits of the fruit.

(Recebido para publicação em 28 de janeiro de 2014; aceito em 7 de janeiro de 2015) (Received on January 28, 2014; accepted on January 7, 2015)

$\mathrm{O}$ morango é um fruto rico em vitamina $C$, possuindo também vitaminas do complexo $\mathrm{B}$, além de cálcio, magnésio, fósforo e ferro (TACO, 2011), sendo consumido principalmente in natura além de industrializado. No Brasil, a estimativa de produção de morango em 2013, foi de 110 mil toneladas, e área cultivada de 4.200 hectares, tendo à frente Minas Gerais, São Paulo e Rio Grande do Sul (Antunes et al., 2014). O estado de São Paulo, nesse mesmo ano, produziu 9.773 toneladas de morango, em 300,4 hectares, distribuídos em 35 municípios, sendo os maiores em área cultivada, Piedade (80 ha), Atibaia (30 ha), Jarinu (25 ha), Campinas (20 ha) e
Itapeva (20 ha) (IEA, 2013).

Do ponto de vista sócio-econômico, o morango é uma espécie importante, sendo que a rentabilidade varia de 50 a $100 \%$ do valor investido, dependendo do custo de produção, inclusive da mão de obra (Antunes et al., 2014). A produção é variável com o genótipo (Oliveira \& Scivittaro, 2006, 2011; Pallamin, 2007; Purquerio et al. 2007; Oliveira et al., 2008; Antunes et al., 2010), região (Radin et al., 2011), sistema de cultivo (Fernandes Júnior \& Leal, 2009; Resende et al., 2010; Camargo et al., 2011), época de plantio (Oliveira et al., 2008; Pereira et al., 2013), ano (Duarte Filho, 2006; Oliveira et al., 2008) e procedên- cia das mudas (Oliveira \& Scivittaro, 2006). Dentre as cultivares, as principais utilizadas em São Paulo são Camarosa, Camino Real, Festival e Oso Grande, desenvolvidas nos Estados Unidos da América, sendo Oso Grande líder em Atibaia e Jarinu e Camino Real em Piedade (Passos et al., 2014). Entretanto, há poucos trabalhos recentes de pesquisa sobre o desempenho de cultivares de morangueiro, objetivando o cultivo nas condições do estado de São Paulo.

O objetivo do presente trabalho foi avaliar o desempenho agronômico das cultivares de morangueiro Aleluia, Camarosa, Camino Real, Festival, Oso Grande, Tudla e Ventana, visando a 
determinação das melhores cultivares em termos de produtividade e qualidade dos frutos.

\section{MATERIAL E MÉTODOS}

O experimento foi instalado em maio de 2008, na Fazenda Santa Elisa do Instituto Agronômico de Campinas, em Campinas-SP (22 $54^{\prime} 20^{\prime \prime}, 47^{\circ} 05^{\prime} 34^{\prime \prime}$, altitude $674 \mathrm{~m}$ ), com clima classificado segundo Köppen, como tipo Cwa, que corresponde ao clima tropical de altitude, com chuvas no verão e seca no inverno, com temperatura média do mês mais quente superior a $22^{\circ} \mathrm{C}$ (Miranda et $a l ., \mathrm{s} / \mathrm{d})$. No período de experimentação, de acordo com o Centro de Ecofisiologia e Biofísica do IAC, foram observadas as seguintes médias de temperaturas máximas $\left({ }^{\circ} \mathrm{C}\right)$ e mínimas $\left({ }^{\circ} \mathrm{C}\right), 24,1 \mathrm{e}$ 13,6 em maio; 24,6 e 13,5 em junho; 26,3 e 12,2 em julho; 27,4 e 14,7 em agosto; 26,6 e 14,7 em setembro e 28,9 e 18,2 em outubro.

O delineamento experimental utilizado foi em blocos ao acaso, com três repetições e sete cultivares de morango (Aleluia, Camino Real, Camarosa, Festival, Oso Grande, Tudla e Ventana). A unidade experimental correspondente a 1,96 $\mathrm{m}^{2}$ foi constituída de 14 plantas, equivalendo a 50.000 plantas/ha, considerando $70 \%$ da área útil como área cultivada.

$\mathrm{O}$ estudo foi conduzido em Latossolo Vermelho Escuro Distrófico (Embrapa, 2006) contendo as seguintes características químicas: matéria orgânica $=25 \mathrm{~g} / \mathrm{dm}^{3} ; \mathrm{pH}\left(\mathrm{em} \mathrm{CaCl}_{2}\right)=5,9 ; \mathrm{P}$ (resina) $=199 \mathrm{mg} / \mathrm{dm}^{3} ; \mathrm{K}, \mathrm{Mg}, \mathrm{Ca}, \mathrm{H}+\mathrm{Al}$ e CTC $=0,55 ; 2,3 ; 5,0 ; 1,6$ e 9,52 $\mathrm{cmol}_{\mathrm{c}} /$ $\mathrm{dm}^{3} ; \mathrm{V}=83 \%$; Fe, Mn, Cu, Zn e B=8,0; 8,$7 ; 4,0 ; 3,3$ e $0,3 \mathrm{mg} / \mathrm{dm}^{3}$.

Aos 60 dias antes do plantio realizou-se calagem com calcário dolomítico $(\mathrm{PRNT}=90 \%)$, distribuindo-se a lanço em área total do terreno. Após isso, o corretivo foi incorporado com uso de enxada rotativa, até $20 \mathrm{~cm}$ de profundidade, irrigando-se a seguir. Os canteiros apresentavam $70 \mathrm{~cm}$ de largura e $20 \mathrm{~cm}$ de altura, com duas linhas distanciadas de $40 \mathrm{~cm}$ ao longo dos mesmos. A adubação de pré-plantio constou da aplicação de $100 \mathrm{~g} / \mathrm{m}^{2}$ da fórmula 08-28-16. A adubação de cobertura foi realizada na forma de nitrato de amônio ( $5 \mathrm{~g} /$ planta) e de adubo fórmula 10-10-10 (20 g/planta), aos 30 e 70 dias após o transplante das mudas, respectivamente. As adubações de plantio e cobertura basearam-se nas recomendações de Passos \& Trani (1996). Farpas de madeira foram aplicadas entre as plantas como cobertura de solo, para proteção dos frutos do contato com o solo, seguindo recomendação de Camargo Filho \& Camargo (2009). Os outros tratos culturais constaram do controle do ácaro rajado, eliminação do mato, folhas secas e estolhos, além de irrigações para manter o solo próximo à capacidade de campo.

As mudas de morangueiro utilizadas foram adquiridas em bandejas plásticas com substrato à base de fibra de coco provenientes de viveiro comercial. As mudas com cerca de 40 dias foram transplantadas para os canteiros definitivos em 14/05/08, no espaçamento de $40 \times 35 \mathrm{~cm}$.

As colheitas de frutos com cerca de $75 \%$ da superfície madura ocorreram de 13/06/08 a 15/10/08, totalizando 29 colheitas, na área útil das parcelas constituída de 10 plantas $\left(1,40 \mathrm{~m}^{2}\right)$. Em cada colheita foi avaliada a massa fresca dos frutos, sendo agrupadas em cinco classes: $\leq 10,0 \mathrm{~g}$ (frutos pequenos, segundo Chandler et al., 2005); 10,1 a 20,0 g; 20,1 a 30,0 g; 30,1 a 40,0 g e 40,1 a 50,0 g, sendo totalizados para o período de junho a julho (de melhores preços) e junho a outubro (período total de colheitas). Isso foi feito, pois a cotação do morango leva em conta o tamanho do mesmo e o pico de safra ocorre em agosto (Camargo Filho \& Camargo, 2009). Foi também calculada a porcentagem de produção de frutos em cada uma das classes mencionadas, para cada uma das cultivares, no período total de colheitas (junho a outubro) e as produções e número de frutos por planta e massa média do fruto $(\mathrm{g})$ nesses dois períodos.

Nos frutos maduros de todas as plantas de cada parcela, congelados $\left(-18^{\circ} \mathrm{C}\right)$ imediatamente após a colheita, foram determinados sólidos solúveis em `Brix (SS), acidez titulável em \% (AT), relação $\mathrm{SS} / \mathrm{AT}$ e teor de vitamina $\mathrm{C}$ em $\mathrm{mg} / 100 \mathrm{~g}$ de massa de fruto, seguindo a metodologia descrita por Carvalho et al. (1990). Os dados de SS, AT e SS/AT se referem à média dos valores obtidos em 13 e 18/08/08, sendo que em cada data foram feitas duas repetições de laboratório. Os dados do teor de vitamina $\mathrm{C}$ dizem respeito a frutos colhidos em 21/08, 27/08 e 24/09/08, respectivamente para a primeira, segunda e terceira repetição de campo; para cada parcela foram feitas duas determinações.

Para a análise estatística dos dados, utilizou-se o teste F e para comparação das médias das cultivares, o teste de Tukey, sendo empregado o programa Sisvar 5.3 Build 77 (Ferreira, 2008). Pelo teste de Hartley, verificou-se que não havia necessidade de transformação dos dados. Foi feita também a correlação entre a produção total, o número total de frutos e a massa média do fruto, e entre a produção total e a produção em cada uma das classes, sendo a significância dos coeficientes de correlação avaliada pelo teste t a $5 \%$ de probabilidade.

\section{RESULTADOS E DISCUSSÃO}

No período inicial de colheitas (junho-julho) não houve diferença entre as cultivares na produção e número de frutos por planta (Tabela 1). Quanto à massa do fruto, ela foi superior para 'Ventana', significando uma vantagem para essa cultivar, devido à relação positiva entre esse caráter e a classificação do morango no mercado (Camargo Filho \& Camargo, 2009).

No período total de colheitas (junho-outubro) 'Ventana', sem diferir de 'Festival' e 'Aleluia', foi a mais produtiva. 'Festival' e 'Ventana', sem diferirem de 'Camarosa' e 'Aleluia', produziram maior número de frutos. 'Aleluia' produziu frutos de maior massa que 'Camino Real', sendo que ambas não diferiram das demais cultivares. A produção por planta correlacionou-se significativa e positivamente com o número de frutos e a massa do fruto, sendo os coeficientes de correlação, respectivamente, de 0,92 e de 0,62 . Isso significa que o número de frutos teve maior influência na produção do que a massa do fruto. Por outro lado, a correlação entre o número de frutos e a massa do fruto não foi significativa, sen- 
Tabela 1. Produção (g/planta), número de frutos por planta e massa fresca do fruto em junho-julho e junho-outubro \{yield (g/plant), number of fruits/plant and fresh fruit mass in June-July and from June to October\}. Campinas, IAC, 2008.

\begin{tabular}{|c|c|c|c|c|c|c|}
\hline \multirow[b]{2}{*}{ Cultivares } & \multicolumn{3}{|c|}{ Junho-julho } & \multicolumn{3}{|c|}{ Junho-outubro } \\
\hline & $\begin{array}{l}\text { Produção } \\
\text { (g/planta) }\end{array}$ & $\begin{array}{c}\text { Frutos/planta } \\
\qquad\left(\mathbf{n}^{0}\right)\end{array}$ & $\begin{array}{l}\text { MF } \\
\text { (g) }\end{array}$ & $\begin{array}{l}\text { Produção } \\
\text { (g/planta) }\end{array}$ & $\begin{array}{c}\text { Frutos/planta } \\
\left(\mathbf{n}^{0}\right)\end{array}$ & $\begin{array}{l}\text { MF } \\
\text { (g) }\end{array}$ \\
\hline Aleluia & $34,6^{\text {ns }}$ & $3,2^{\mathrm{ns}}$ & $11,0 \mathrm{a}$ & 221,2 bcd & $17,2 \mathrm{ab}$ & $13,0 \mathrm{~b}$ \\
\hline Camarosa & 51,3 & 4,7 & $11,0 \mathrm{a}$ & $181,4 \mathrm{abc}$ & $17,3 \mathrm{ab}$ & $10,5 \mathrm{ab}$ \\
\hline Camino Real & 37,0 & 3,8 & $9,7 \mathrm{a}$ & $121,4 \mathrm{a}$ & $12,3 \mathrm{a}$ & $9,8 \mathrm{a}$ \\
\hline Festival & 65,7 & 5,6 & $12,0 \mathrm{a}$ & $244,4 \mathrm{~cd}$ & $23,4 \mathrm{~b}$ & $10,5 \mathrm{ab}$ \\
\hline Oso Grande & 38,0 & 2,9 & $12,9 \mathrm{a}$ & $142,2 \mathrm{ab}$ & $13,1 \mathrm{a}$ & $10,8 \mathrm{ab}$ \\
\hline Tudla & 51,9 & 4,6 & $11,4 \mathrm{a}$ & $149,0 \mathrm{ab}$ & $14,4 \mathrm{a}$ & $10,4 \mathrm{ab}$ \\
\hline Ventana & 68,6 & 3,4 & $20,4 \mathrm{~b}$ & $301,3 \mathrm{~d}$ & $24,1 \mathrm{~b}$ & $12,4 \mathrm{ab}$ \\
\hline CV (\%) & 27,8 & 28,0 & 12,4 & 17,2 & 13,8 & 9,3 \\
\hline
\end{tabular}

Médias com a mesma letra na coluna não diferem pelo teste de Tukey, $5 \%$ (means followed by the same letter in the column are not different by Tukey test, $5 \%$ ). ns = não significativo (not significant).

Tabela 2. Produção (g/planta) e porcentagem de frutos referentes a três classes de massa fresca no período de junho a outubro \{yield (g/ plant) and percentage of fruits concerning three classes of fresh mass from June to October). Campinas, IAC, 2008.

\begin{tabular}{|c|c|c|c|c|c|c|}
\hline \multirow{2}{*}{ Cultivares } & \multicolumn{3}{|c|}{ Produção (g/planta) } & \multicolumn{3}{|c|}{ Produção (\%) } \\
\hline & $\leq 10 \mathrm{~g}$ & $10,1-20 \mathrm{~g}$ & 20,1-30 g & $\leq 10 \mathrm{~g}$ & $10,1-20 \mathrm{~g}$ & $20,1-30 \mathrm{~g}$ \\
\hline Aleluia & $48,0 \mathrm{ab}$ & $108,8 \mathrm{ab}$ & $53,6 \mathrm{~b}$ & 22,0 & $49,3 \mathrm{ab}$ & $23,7 \mathrm{~b}$ \\
\hline Camarosa & $68,4 \mathrm{abc}$ & $82,0 \mathrm{a}$ & $30,0 \mathrm{ab}$ & $38,0^{\mathrm{ns}}$ & $45,1 \mathrm{a}$ & $16,2 \mathrm{ab}$ \\
\hline Camino Real & $48,2 \mathrm{ab}$ & $68,8 \mathrm{a}$ & $4,5 \mathrm{a}$ & 40,2 & $56,5 \mathrm{~b}$ & $3,4 \mathrm{a}$ \\
\hline Festival & $80,1 \mathrm{c}$ & $135,3 \mathrm{~b}$ & $25,8 \mathrm{ab}$ & 32,7 & $55,3 \mathrm{ab}$ & $10,6 \mathrm{ab}$ \\
\hline Oso Grande & $40,3 \mathrm{a}$ & $82,0 \mathrm{a}$ & $18,9 \mathrm{ab}$ & 28,9 & $57,4 \mathrm{~b}$ & $13,2 \mathrm{ab}$ \\
\hline Tudla & $49,7 \mathrm{abc}$ & $75,3 \mathrm{a}$ & $20,9 \mathrm{ab}$ & 33,6 & $50,7 \mathrm{~b}$ & $13,7 \mathrm{ab}$ \\
\hline Ventana & $71,6 \mathrm{bc}$ & $148,9 \mathrm{~b}$ & $52,3 \mathrm{~b}$ & 24,2 & $49,7 \mathrm{ab}$ & $17,5 \mathrm{ab}$ \\
\hline $\mathrm{CV}(\%)$ & 18,8 & 17,8 & 44,2 & 20,5 & 6,9 & 39,2 \\
\hline
\end{tabular}

Médias com a mesma letra na coluna não diferem pelo teste de Tukey, $5 \%$ (means followed by the same letter in the column are not different by Tukey test, $5 \%$ ). ns = não significativo (not significant).

do encontradas cultivares com diversas combinações dessas variáveis.

A comparação da produtividade entre as cultivares Camarosa, Oso Grande e Tudla concorda com Duarte Filho (2006) em trabalho também realizado em condições de campo, em Caldas-MG, e com Radin et al. (2011), em ambiente protegido, em Caxias do Sul e Eldorado do Sul-RS. Na comparação entre as cultivares Camarosa e Oso Grande houve concordância com Duarte Filho (2006) e Pallamin (2007), também em condições de campo, respectivamente em Caldas-MG, e Bauru-SP, e com Purquerio et al. (2007), Radin et al. (2011) e Costa et al. (2011), em ambiente protegido, respectivamente em Socorro-SP, Eldorado do Sul e Caxias do Sul-RS, e
Passo Fundo-RS. Ainda, na comparação entre as cultivares Camarosa e Oso Grande houve concordância com Resende et al. (2010) em Guarapuava-PR, em ambiente protegido e discordância com esses autores em condições de campo. $\mathrm{Na}$ comparação entre as cultivares Festival e Camarosa os resultados são discordantes de Antunes et al. (2010), em ambiente protegido, em Pelotas-RS. As produtividades obtidas no presente trabalho ficaram abaixo do relatado por Duarte Filho (2006), Pallamin (2007), Purquerio et al. (2007), Antunes et al. (2010), Resende et al. (2010) e Costa et al. (2011), mas se aproximaram dos valores obtidos por Radin et al. (2011). No caso de 'Oso Grande' houve concordância do valor da produtividade com
Fernandes Júnior \& Leal (2009) em cultivo vertical realizado em Campinas-SP, em julho, e também, se aproximou dos dados de Zorzeto (2011), também em cultivo em substrato (sem solo) em ambiente protegido, realizado em Jundiaí-SP, em julho. É possível, que tenha havido influência da época de plantio, pois as médias das temperaturas máximas $\left(24,1^{\circ} \mathrm{C}\right)$ e mínimas $\left(13,6^{\circ} \mathrm{C}\right)$ em maio já estavam dentro da faixa favorável à floração e frutificação (13-26 $\left.{ }^{\circ} \mathrm{C}\right)$, de acordo com Passos et al. (2014). Esses autores recomendam o plantio em março-abril, nas regiões tradicionalmente produtoras de morango em São Paulo. O fraco desempenho de 'Camino Real' no presente trabalho nas condições de Campinas, sugere que ela 
Tabela 3. Teores de sólidos solúveis (SS, ${ }^{\circ}$ Brix), acidez titulável (AT, \%), relação SS/AT e de vitamina $\mathrm{C}$ ( $\mathrm{mg} / 100 \mathrm{~g}$ massa) de morangos maduros de sete cultivares \{soluble solids (SS, ${ }^{\circ}$ Brix), titratable acidity (AT, \%), SS/AT ratio and vitamin C content (mg/100 g mass) of ripe strawberries of seven cultivars $\}$. Campinas, IAC, 2008.

\begin{tabular}{lccccc}
\hline Cultivares & SS $^{\mathbf{1}}$ & AT $^{\mathbf{1}}$ & SS/AT $^{\mathbf{1}}$ & Vit. C $^{\mathbf{2}}$ \\
\hline Aleluia & $8,10 \mathrm{~b}$ & $0,84 \mathrm{bc}$ & $9,63 \mathrm{c}$ & 68,03 \\
Camarosa & $7,50 \mathrm{ab}$ & $0,92 \mathrm{c}$ & $8,10 \mathrm{ab}$ & 65,30 \\
Camino Real & $6,63 \mathrm{a}$ & $0,78 \mathrm{ab}$ & $8,50 \mathrm{abc}$ & 67,90 \\
Festival & $7,73 \mathrm{~b}$ & $0,80 \mathrm{ab}$ & $9,60 \mathrm{c}$ & 74,80 \\
Oso Grande & $7,07 \mathrm{ab}$ & $0,75 \mathrm{a}$ & $9,37 \mathrm{bc}$ & 56,03 \\
Tudla & $6,50 \mathrm{a}$ & $0,89 \mathrm{c}$ & $7,23 \mathrm{a}$ & 66,00 \\
Ventana & $7,13 \mathrm{ab}$ & $0,79 \mathrm{ab}$ & $8,97 \mathrm{bc}$ & 66,70 \\
\hline CV (\%) & 5,14 & 3,58 & 5,36 & 10,72 \\
\hline
\end{tabular}

${ }^{1}$ Médias com a mesma letra na coluna não diferem entre si pelo teste de Tukey, $5 \%$ (means followed by the same letter in the column are not different by Tukey test, 5\%). ns= não significativo (not significant). ${ }^{1}$ média das colheitas de 13 e 18/08/08 (mean of August 13 and 18 harvests). ${ }^{2}$ média das colheitas de 21/08, 27/08 e 24/09/08 (mean of August 21 and 27 and September 24 harvests).

possui maior exigência em frio, haja vista que essa cultivar é a mais utilizada em Piedade-SP, segundo Passos et al. (2014).

As cultivares Festival, Oso Grande, Tudla e Ventana (Tabela 1), especialmente esta última, seguiram o comportamento normal das cultivares de morangueiro, que apresentam frutos de maior massa no início das colheitas, segundo Leis et al. (2002), citados por Oliveira et al. (2008). 'Camarosa' e 'Camino Real' praticamente mantiveram a mesma massa do fruto ao longo do ciclo de cultivo. Por outro lado, 'Aleluia' comportou-se de modo distinto, apresentando frutos com menos massa no período inicial de colheitas do que no total. As massas médias dos frutos de 'Ventana' e 'Camarosa' obtidas neste trabalho se aproximaram e foram inferiores, respectivamente, ao obtido por Oliveira \& Scivittaro (2011), em Pelotas-RS. No caso das cultivares Camino Real e Camarosa as massas dos frutos foram inferiores ao verificado por Oliveira et al. (2008), em Pelotas. As massas dos frutos de 'Camarosa', 'Oso Grande' e 'Tudla' foram próximas do obtido por Duarte Filho (2006), em Caldas-MG. As massas dos frutos de 'Camarosa' e 'Oso Grande' foram próximas do obtido por Resende et al. (2010) e Costa et al. (2011) e no caso de 'Camarosa' e 'Festival' inferiores ao verificado por Antunes et al. (2010). Isso sugere que o comportamento das cultivares de morangueiro é variável e dependente das condições de cultivo.

Comparando-se as cultivares nas três classes de massa de frutos mais representativas, no período de junho-outubro (Tabela 2), 'Ventana' e 'Festival' situaram-se no grupo das mais produtivas nas três classes, enquanto 'Aleluia' situou-se no grupo das mais produtivas nas classes de frutos de maior massa. 'Camarosa'e 'Tudla' situaram-se no grupo das mais produtivas de frutos pequenos $(\leq 10 \mathrm{~g})$ e de frutos de maior massa. Desse modo, com exceção de 'Camino Real', as demais cultivares apresentaram potencial para produção de frutos graúdos. Quanto à distribição percentual da produção, 'Camino Real' apresentou $96,7 \%$ de produção nas classes de frutos pequenos e de 10,1 a 20,0 g, enquanto as demais cultivares apresentaram produção distribuída nas três classes. Como os frutos maiores alcançam melhores preços no mercado há vantagens para as cultivares Aleluia e Ventana. Essas cultivares também produziram frutos com massa acima de 30 g. Houve correlação significativa e positiva entre a produção por planta e as produções das três classes predominantes, sendo os coeficientes de correlação respectivamente de 0,62 para a classe de frutos pequenos, 0,95 para a classe de frutos de 10,1 a $20,0 \mathrm{~g}$ e de 0,73 para a classe de frutos de 20,1 a 30,0 g.
Quanto à qualidade, 'Aleluia' e 'Festival' apresentaram maior teor de sólidos solúveis que 'Camino Real' e 'Tudla', sem diferirem das demais (Tabela 3). 'Camarosa' e 'Tudla', sem diferirem de 'Aleluia', apresentaram maior acidez titulável que as demais. 'Aleluia' e 'Festival', sem diferirem de 'Oso Grande', 'Ventana' e 'Camino Real', apresentaram as maiores relações entre os teores de sólidos solúveis e de acidez titulável. Não houve diferença significativa entre as cultivares, quanto ao teor de vitamina $C$. Esses resultados são concordantes com os de Purquerio et al. (2007), para os valores superiores de 'Aleluia', 'Festival' e 'Oso Grande' para o teor de sólidos solúveis e da relação SS/AT, mas houve discordância quanto ao teor de vitamina C. De acordo com Chitarra \& Chitarra (2005), citados por Camargo et al. (2011), para o morango, comercialmente o conteúdo mínimo de sólidos solúveis é de 7,0\% e o máximo de acidez titulável é $0,8 \%$, resultando no valor 8,75 entre ambos, sendo o melhor balanço entre os constituintes de doçura e acidez. No presente trabalho, apenas as cultivares Aleluia, Festival, Oso Grande e Ventana apresentaram valores dessa relação superiores a 8,75. Os resultados obtidos para essa relação para 'Camino Real', 'Oso Grande', 'Tudla' e 'Ventana' por Camargo et al. (2011) são concordantes com os do presente trabalho. O teor de vitamina $\mathrm{C}$ obtido neste trabalho mostrou-se superior ao de Pallamin (2007) e também superior ao obtido por Purquerio et al. (2007) para 'Camarosa', 'Festival' e 'Oso Grande' e semelhante para 'Aleluia'. No presente trabalho os teores de vitamina $\mathrm{C}$ ficaram situados na faixa de 56,03 a $74,80 \mathrm{mg} / 100 \mathrm{~g}$ de massa fresca não diferindo entre si, podendo ser considerados normais de acordo com TACO (2011), que cita 63,6 como valor de referência.

As sete cultivares avaliadas apresentaram diferenças significativas quanto à produtividade e seus componentes e qualidade do fruto. 'Ventana', sem diferir de 'Aleluia' e 'Festival', pode ser considerada a cultivar mais produtiva, inclusive com potencial para produção de frutos graúdos. 'Aleluia', 'Festival', 'Oso Grande' e 'Ventana' constituem o grupo de melhor relação entre sólidos solúveis e acidez titulável. Assim, ‘Ale- 
luia' e 'Ventana' são alternativas para o cultivo do morangueiro no estado de São Paulo.

\section{REFERÊNCIAS}

ANTUNES LEC; VIGNOLO GK; GONÇALVES MA. 2014. Morango mostra tendência de crescimento de mercado. In: Campo \& Negócios, Anuário HF p.54-57.

ANTUNES LEC; RISTOW NC; KROLOW ACR; CARPENEDO S; REISSER JÚNIOR C. 2010. Yield and quality of strawberry cultivars. Horticultura Brasileira 28: 222-226.

CAMARGO FILHO WP; CAMARGO FP. 2009. Análise da produção de morango dos estados de São Paulo e Minas Gerais e do mercado da CEAGESP. Informações Econômicas 39: 42-50.

CAMARGO LKP; RESENDE JTV; TOMINAGA TT; KURCHAIDT SM; CAMARGO CK; FIGUEIREDO AST. 2011. Postharvest quality of strawberry fruits produced in organic and conventional systems. Horticultura Brasileira 29: 577-583.

CARVALHO CRL; MANTOVANI DMB; CARVALHO PRN; MORAES RM. 1990. Analises químicas de alimentos. Campinas: Instituto de Tecnologia de Alimentos. 121p (Manual Técnico).

CHANDLER CK; SUMLER JR. JC; RONDON SI. 2005. Evaluation of strawberry cultivars grown under a high plastic tunnel in West Central Florida. Proc. Fla. State Hort.Soc. 118: 113-114.

COSTA RC; CALVETE EO; REGINATTO FH; CECCHETTI D; LOSS JT; RAMBO A; TESSARO F. 2011. Telas de sombreamento na produção de morangueiro em ambiente protegido. Horticultura Brasileira 29: 98-102.

DUARTE FILHO J. 2006. Cultivares de morango. In: CARVALHO SP (coord). Boletim do Morango: cultivo convencional, segurança alimentar, cultivo orgânico. Belo Horizonte: FAEMG, p.15-22.

EMBRAPA. 2006. Centro Nacional e Pesquisa em Solos. Sistema Brasileiro de Classificação de Solos. Brasilia: Embrapa-SPI; Rio de Janeiro: Embrapa-Solos. 306p.

FERNANDES JÚNIOR F; LEAL PAM. 2009. Radiação fotossinteticamente ativa em cultivo vertical de morangueiros em função do espaçamento e superfície refletora. Horticultura Brasileira 27: S3177-S3183 (Suplemento - CD ROM).

FERREIRA DF. 2008. SISVAR: um programa para análises e ensino de estatística. Revista Symposium 6: 36-41.

IEA. INSTITUTO DE ECONOMIAAGRÍCOLA. 2013. Morango. In: Área e produção dos principais produtos da agropecuária do estado de São Paulo. Disponível em http://www.iea. sp.gov.br/out/bancodedados.html. Acessado em 25/08/2014.

MIRANDA MJ; PINTO HS; ZULLO JÚNIOR J; FAGUNDES RM; FONSECHI DB; CALVE L; PELLEGRINO GQ. Clima dos Municípios Paulistas. CEPAGRI Meteorologia UNICAMP. Disponível em http://www.cpa. unicamp.br/outras-informacoes/clima-dosmunicipios. Acessado em 24/10/2012.

OLIVEIRA RP; SCIVITTARO WB. 2006. Desempenho produtivo de mudas nacionais e importadas de morangueiro. Revista Brasileira de Fruticultura 28: 520-522.

OLIVEIRA RP; SCIVITTARO WB. 2011. Desempenho produtivo de cultivares de morangueiro. Scientia Agraria 12: 69-74.

OLIVEIRA RP; SCIVITTARO WB; FINKENAUER D. 2008. Produção de morangueiro da cv. Camino Real em sistema de túnel. Revista Brasileira de Fruticultura 30: 681-684.

PALLAMIN ML. 2007. Alternativas no controle fitossanitário em diferentes cultivares de morangueiro como ferramenta na produção integrada. Botucatu: UNESP. 60p. (Dissertação mestrado).
PASSOS FA; TRANI PE. 1996. Morango. In: RAIJ B; CANTARELLA H; QUAGGIO JA; FURLANI AMC (eds) Recomendações de adubação e calagem para o estado de São Paulo. $2^{\mathrm{a}}$ ed. Campinas, Instituto Agronômico \& Fundação IAC, p.182 (Boletim técnico, $100)$.

PASSOS FA; TRANI PE; SANCHES J; ANTONIALI S; WATANABE AT; SEMIS JB; SALOMON MV; BORZACCHINI O. 2014. Morango. In: AGUIAR ATE; GONÇALVES C; PATERNIANI MEAGZ; TUCCI MLS; CASTRO CEF (eds) Instruções agrícolas para as principais culturas econômicas. $7^{\mathrm{a}} \mathrm{ed}$. rev. e atual. Campinas: Instituto Agronômico. p. 283-287 (Boletim IAC, $\mathrm{n}^{\circ}$ 200).

PEREIRAWR; SOUZARJ; YURI JE; FERREIRA S. 2013. Produtividade de cultivares de morangueiro, submetidas a diferentes épocas de plantio. Horticultura Brasileira 3: 500-503.

PURQUERIO LFV; PASSOS FA; TIVELLI SW; TURCO PHN; MAININE C; CARVALHO CRL. 2007. Produtividade e qualidade de frutos de morango de seis cultivares em Socorro-SP. Horticultura Brasileira 25 (Suplemento-CD ROM).

RADIN B; LISBOA BB; WITTER S; BARNI V; REISSER JUNIOR C; MATZENAUER R; FERMINO MH. 2011. Desempenho de quatro cultivares de morangueiro em duas regiões ecoclimáticas do Rio Grande do Sul. Horticultura Brasileira 29: 287-291.

RESENDE JTV; MORALES RGF; FARIA MV; RISSINI ALL; CAMARGO LKP; CAMARGO CK. 2010. Produtividade e teor de sólidos solúveis de frutos de cultivares de morangueiro em ambiente protegido. Horticultura Brasileira 28: 185-189.

TACO. 2011. Tabela brasileira de composição de alimentos. NEPA-UNICAMP. 4. ed. rev. e ampl. Campinas: NEPA-UNICAMP. 161p.

ZORZETO TQ. 2011. Caracterização física e química de substratos para plantas e sua avaliação no rendimento do morangueiro (Fragaria x ananassa). Campinas: IAC. 96p. (Dissertação mestrado). 